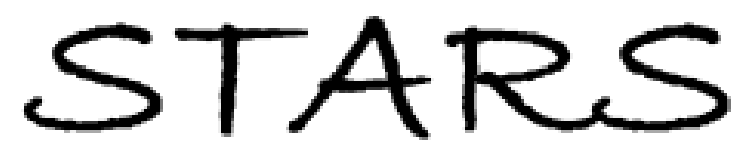

University of Central Florida

STARS

Faculty Bibliography 2000s

Faculty Bibliography

$1-1-2009$

\title{
Extreme Value Statistics in Silicon Photonics
}

D. Borlaug

S. Fathpour

University of Central Florida

B. Jalali

Find similar works at: https://stars.library.ucf.edu/facultybib2000

University of Central Florida Libraries http://library.ucf.edu

This Article is brought to you for free and open access by the Faculty Bibliography at STARS. It has been accepted for inclusion in Faculty Bibliography 2000s by an authorized administrator of STARS. For more information, please contactSTARS@ucf.edu.

\section{Recommended Citation}

Borlaug, D.; Fathpour, S.; and Jalali, B., "Extreme Value Statistics in Silicon Photonics" (2009). Faculty Bibliography 2000s. 1366.

https://stars.library.ucf.edu/facultybib2000/1366

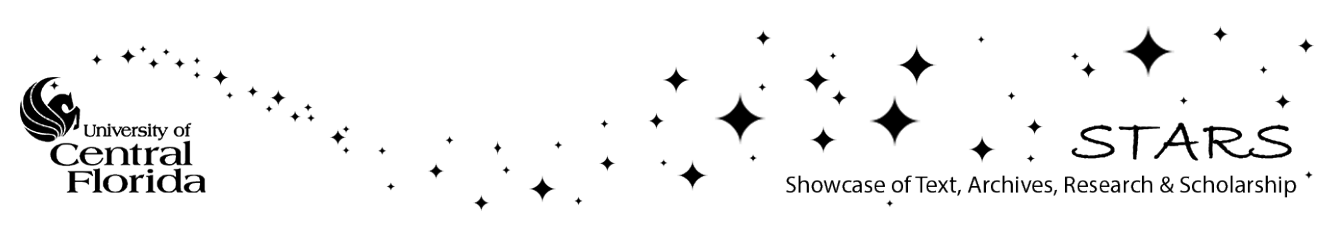




\section{IEEE Photonics Journal \\ An IEEE Photonics Society Publication}

\section{Extreme Value Statistics in Silicon Photonics}

Volume 1, Number 1, June 2009

D. Borlaug

S. Fathpour, Member, IEEE

B. Jalali, Fellow, IEEE

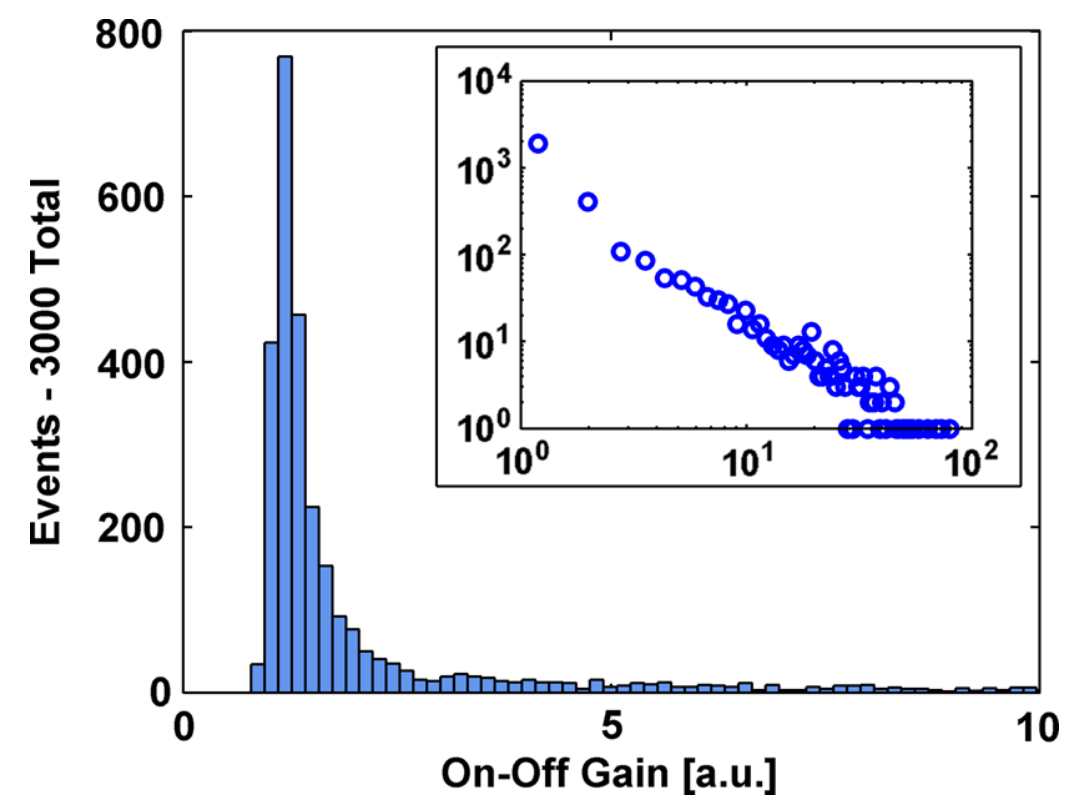

DOI: 10.1109/JPHOT.2009.2025517

1943-0655/\$25.00 @2009 IEEE 


\title{
Extreme Value Statistics in Silicon Photonics
}

\author{
D. Borlaug, ${ }^{1}$ S. Fathpour, ${ }^{1,2}$ Member, IEEE, \\ and B. Jalali, ${ }^{1}$ Fellow, IEEE
}

\author{
${ }^{1}$ Electrical Engineering Department, University of California, Los Angeles, CA 90095 USA \\ ${ }^{2}$ CREOL, The College of Optics \& Photonics, University of Central Florida, Orlando, FL 32816 USA \\ DOI: 10.1109/JPHOT.2009.2025517 \\ 1943-0655/\$25.00 @2009 IEEE
}

\begin{abstract}
Manuscript received May 26, 2009; revised June 8, 2009. First published Online June 16, 2009. Current version published July 3, 2009. This work was supported by Dr. Henryk Temkin of DARPA-MTO. Corresponding author: B. Jalali (e-mail: jalali@ucla.edu).
\end{abstract}

\begin{abstract}
L-shape probability distributions are extremely non-Gaussian functions that have been surprisingly successful in describing the occurrence of extreme events ranging from stock market crashes, natural disasters, structure of biological systems, fractals, and optical rogue waves. We show that fluctuations in stimulated Raman scattering, as well as in coherent anti-Stokes Raman scattering, in silicon can follow extreme value statistics and provide mathematical insight into the origin of this behavior.
\end{abstract}

Index Terms: Extreme value statistics, L-shape probability distribution, Raman scattering, silicon photonics.

\section{Introduction}

Extreme value theory is a branch of statistics that describe frequent occurrence of extreme deviations from the median. One class of extreme value distributions are L-shape probability density functions, a set of power-law distributions in which events much larger than the mean (outliers) can occur with significant probability. In stark contrast, the ubiquitous Gaussian distribution heavily favors events close to the mean, and all but forbids highly unusual and cataclysmic events. Extreme value statistics have recently been observed during generation of optical Rogue waves, a soliton-based effect that occurs in optical fibers [1], [2] and during Raman amplification in optical fibers [3], [4]. Here, we experimentally show that the distribution of Raman amplified pulses in silicon, as well as that of coherent anti-Stokes Raman scattering (CARS), in the presence of a noisy pump, follows similar power-law statistics. We describe a model that shows how such unusual distributions emerge from the interaction of random fluctuations with a strongly nonlinear response function. We first begin by discussing previous works, then present our experimental observations, following is a detailed mathematical model describing the extreme occurrences observed, and finally we conclude.

There have been a number of studies on noise in stimulated Raman scattering (SRS) using silicon waveguides [5] and optical fibers. These studies have primarily focused on the role of quantum fluctuations in the amplified spontaneous emission in fibers [6]-[11]. The effects of dispersion and walk-off on the noise characteristics of Stokes pulses in fibers have been studied and a slight skew toward the low energy tail of the distribution was observed in simulations [10]. Shaping of spontaneous emission noise by Raman amplification in fibers has been observed where it was shown that the high energy tail of the distribution is actually suppressed due to initiation of 2nd-order Raman scattering [11]. An enhancement of the high-energy tail of the distribution for 


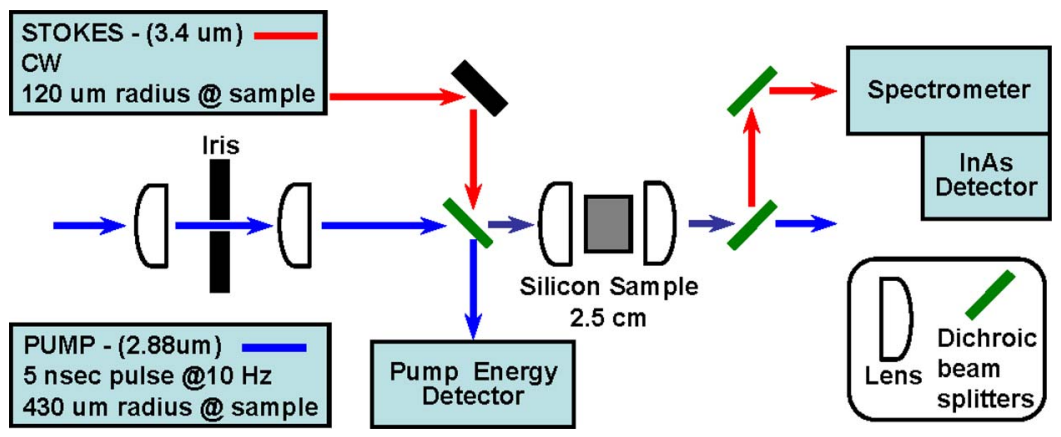

Fig. 1. The experimental setup.

amplified spontaneous emission in optical fibers has been reported [12], however, no connection with extreme value statistics was made until most recently [3].

SRS in silicon has been extensively pursued as a means to create optically pumped amplifiers and lasers to bypass the material's indirect band structure [13]. The technology is particularly promising in the mid-infrared (mid-IR) where two-photon absorption, a competing process that also creates free carrier absorption, is absent. In this paper, we describe the first study of the statistics of Raman amplification in silicon. In experiments performed in the mid-IR, we show how L-shape distributions arise from the Raman amplification of an input Stokes beam by a noisy pump [14].

\section{Experiments and Comparison With Theory}

The experiments were performed in the mid-IR using a $2.5 \mathrm{~cm}$ bulk silicon sample, Fig. 1. A bulk silicon sample was used as opposed to silicon on insulator (SOI) waveguides due to the high loss of $\mathrm{SiO}_{2}$ at mid-IR wavelengths. However, the observance of extreme value behavior arises due to the interplay of pump laser fluctuations with the nonlinear transfer function of the Raman process, hence it is a general characteristics of stimulated Raman amplification with a noisy pump. The pump laser was a Q-switched Nd:YAG pumped optically pumped oscillator (OPO) emitting pulses at a wavelength of 2.9 micrometers. The pulse width, as reported by the manufacturer, was approximately $5 \mathrm{~ns}$ (FWHM), but it is known that the pulse envelope has random intra-pulse structure that changes from pulse to pulse due to multiple longitudinal OPO modes. The Stokes was a continuous-wave HeNe laser at 3.4 micrometers. After travelling through the sample, the Stokes and pump beams are separated using a dichroic beam splitter. Further isolation between the two beams is achieved using a spectrometer. The pump wavelength was detected using a photodetector (Coherent J25LP-MB) with a response time of 80 microseconds. The Stokes wavelength was detected using an InAs photodetector (Judson J12-18C-R01M) with a response time of $15 \mathrm{~ns}$. The detectors were not fast enough to resolve the intra-pulse structure of the envelope, however, this is of no concern because we are merely interested in pulse-to-pulse energy fluctuations for which the detectors are adequate. The detected pump and Stokes signals are then measured using an oscilloscope which is triggered at the pump repetition rate. The oscilloscope waveforms are recorded on a remote laptop running LabView.

Fig. 2 shows a histogram of 3000 recorded pump pulses measured before the silicon sample. As can be seen, the distribution is nearly symmetric around a well-defined mean energy. In other words, it has the general features of a mean centric distribution, such as a Gaussian or Rician distribution. The Raman gain experienced by the Stokes signal is simultaneously obtained by comparing the output Stokes signal in the presence and absence of the pump. Again, only relative pulse-to-pulse statistical fluctuations are of interest here. The histogram (3000 pulses) of the correlated Stokes gain is shown in Fig. 3.

The observed distribution clearly shows L-shape extreme value behavior, highlighted by the high probability of large outliers in the "fat-tail" of the distribution. While most pulses experience modest gain, a small percentage of pulses experience amplification far larger than that of average events. This is even more evident in the log-scale plot shown in the inset. The distribution shows that the 


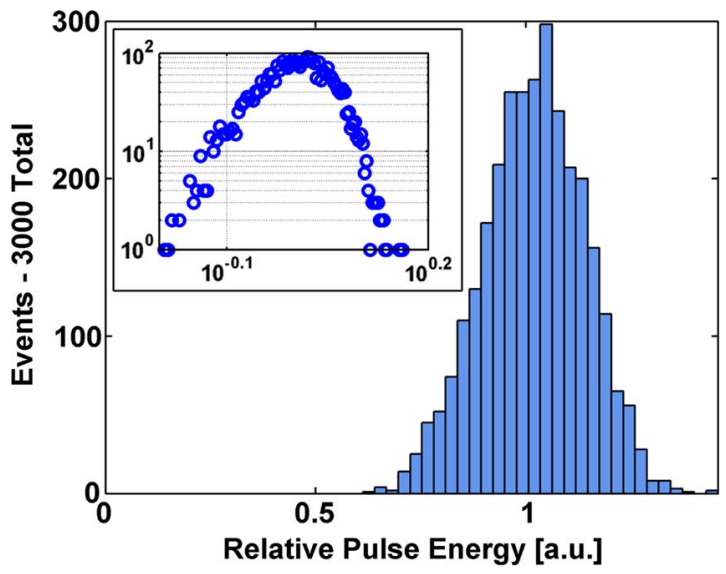

Fig. 2. Measured histogram of pump pulses. Inset shows the same data plotted on a log-log scale.

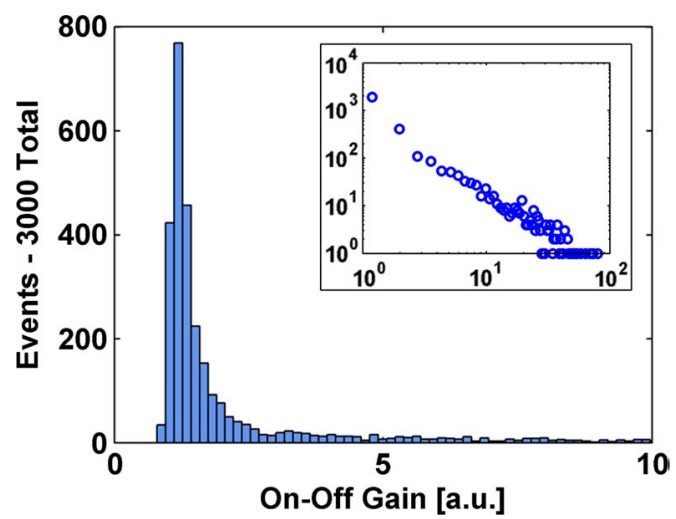

Fig. 3. Measured histogram of amplified Stokes pulses. Inset shows the same data plotted on a log-log scale. Extreme value statistical behavior and outlier events are clearly evident in the tail of the L-shape distribution, easily apparent in the linear nature of the log-log inset.

number of such pulses does not decay exponentially with their amplitude, as would be expected for a Gaussian distribution, but rather has a power-law dependence, as expected for an extreme value random process.

It has previously been shown that the Rician distribution [15] is the proper model for amplitude fluctuations arising from a coherent field perturbed by narrowband noise [16], [17]. We invoke it here as an approximation to fluctuations of the pump laser. Mathematically, the Rician distribution is given by,

$$
f\left(E_{p}\right)=\frac{E_{p}}{\sigma^{2}} \operatorname{Exp}\left[-\frac{E_{p}^{2}+v^{2}}{2 \sigma^{2}}\right] I_{0}\left[\frac{E_{p} v}{\sigma^{2}}\right]
$$

where $E_{p}$ is the electric field amplitude for the pump laser, $I_{0}$ is the modified Bessel function of the first kind with order zero, $v$ and $\sigma^{2}$ are parameters that influence the offset and width of the distribution, respectively. The function approaches a Rayleigh distribution in the limit when $v \rightarrow 0$, evident in Fig. 4. Notice the semblance of Fig. 4 to that of Fig. 2.

Using a common statistical transformations applied with basic electromagnetism, the corresponding pump intensity distribution is given by

$$
f\left(I_{p}\right)=\frac{Z_{0}}{4 n \sigma^{2}} \operatorname{Exp}\left[-\frac{Z_{0} I_{p} / 2 n+v^{2}}{2 \sigma^{2}}\right] I_{0}\left[\frac{v}{\sigma^{2}} \sqrt{\frac{Z_{0} I_{p}}{2 n}}\right] .
$$




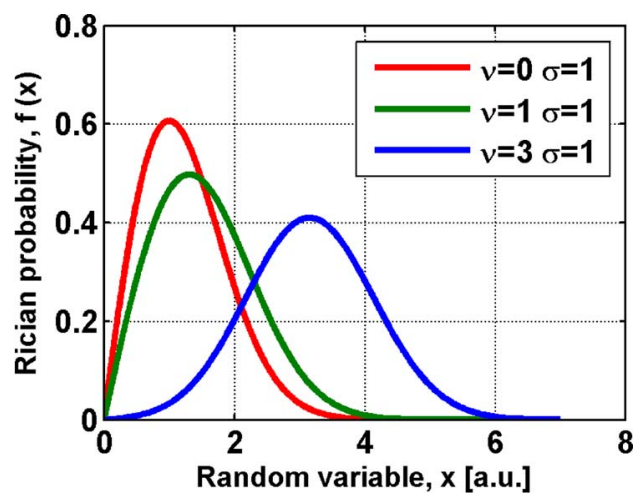

Fig. 4. The Rician probability density function for three sets of input parameters. The Rician distribution is a mean centered distribution but has zero probability of being less than zero-characteristic of electric field amplitude coefficients.

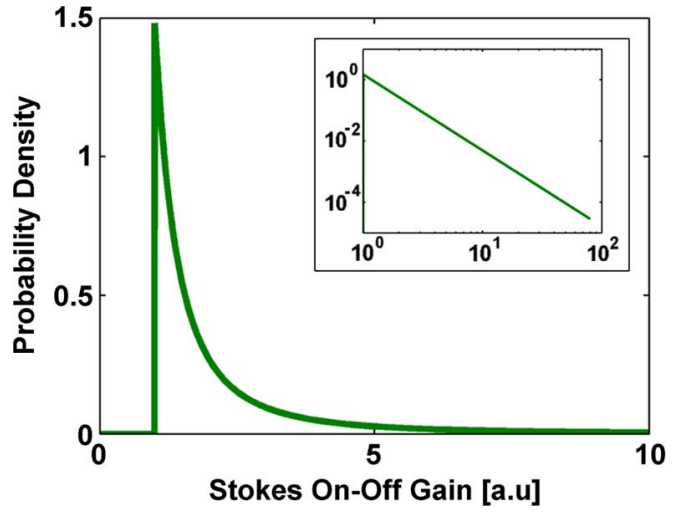

Fig. 5. Distribution of amplified Stokes pulses according to the model in Equation (4). Inset shows the same plotted on the log scale. The model produces the salient features of experimental data shown in Fig. 3.

Here, $I_{p}=2 n E_{p}^{2} / Z_{0}$, where $Z_{0}$ is the impedance of free space and $n$ is the refractive index of the medium.

To show how extreme value behavior emerges from the seemingly innocuous Rician distribution we start with the simple expression for the Raman gain,

$$
G=e^{g \cdot l_{p} \cdot L} .
$$

Here, $g_{R}$ is the Raman gain coefficient and $L$ is the pump-Stokes interaction length. This nonlinear function causes the pump intensity fluctuations, given by Equation (2), to be transformed into the following distribution for the Raman gain,

$$
f(G)=G^{-\left(1+b / 2 \sigma^{2}\right)} \cdot\left(\frac{b}{2 \sigma^{2}} \operatorname{Exp}\left[-\frac{v^{2}}{2 \sigma^{2}}\right]\right) \cdot I_{0}\left[\frac{v \sqrt{b L n(G)}}{\sigma^{2}}\right]
$$

where $b \equiv Z_{0} / 2 n g_{R} L$. The first term in this expression readily predicts that the Raman gain will have a power-law behavior, the hallmark of extreme value statistics. This is graphically shown in the plot of Equation (4) shown in Fig. 5.

The inability to measure the pulse envelope and its intra-pulse structure limits the measurement to that of energy fluctuations, whereas the model is based on the intensity fluctuations. This prevents the quantitative comparison between the experiment and the model. Direct comparison could be made by using a high-speed photodetector capable of capturing the temporal noise bandwidth (unavailable in the mid-IR) or by obtaining experimental or first principle noise 


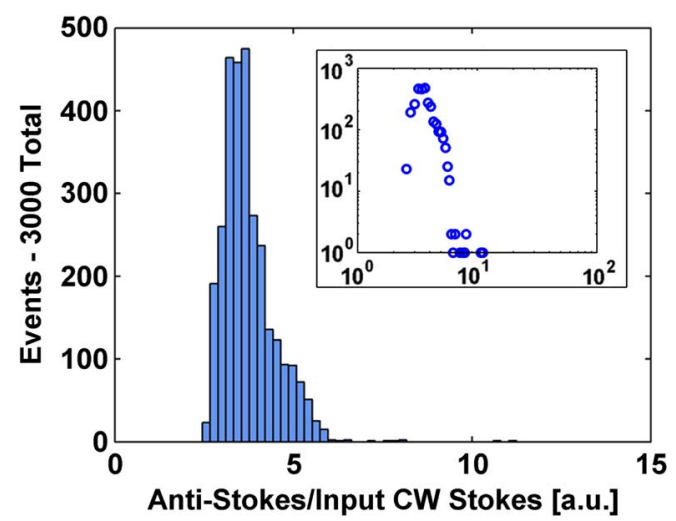

Fig. 6. Measured histogram of anti-Stokes pulses. Inset shows the same data plotted on the log scale.

characteristics and extending the analysis. However, the model unambiguously explains the salient features of the experimental observations. Furthermore, it has recently been shown that rogue wave extreme events observed during supercontinuum generation exhibit qualitatively similar features using either energy or intensity detection [18]. This paper's model shows how extreme value statistics exhibited by the Stokes emerge from transformation of the normal pump intensity fluctuations through the nonlinear relation between gain and pump intensity. Although the Rician distribution was chosen to describe the pump fluctuations, this choice is not critical. A similar formalism can be used to transform other classes of distributions that are operated on by strong nonlinearities, such as a Gaussian transformed into a lognormal distribution; the transformed distribution will exhibit qualitatively similar extreme value behavior.

The same experimental setup, as shown in Fig. 1, is used to measure the statistics of anti-Stokes pulses produced through CARS (a parametric process where Stokes photons are converted to the anti-Stokes wavelength through the Raman nonlinearity). The spectrometer in Fig. 1 is tuned to the anti-Stokes wavelength and the same data acquisition method is used. The anti-Stokes signal is compared to the CW stokes signal entering the gain medium. The histogram of $3000 \mathrm{CARS}$ events is shown in Fig. 6. No attempt was made to create phase matching, therefore the conversion efficiency is expected to be low. The anti-Stokes distribution also has extreme value behavior as evidenced by a clear skew toward high-energy pulses. However, the extent of the high-energy tail of the distribution is less than that observed for Stokes pulses. This can be attributed to the fact that the low conversion efficiency effectively reduces the extent of the extreme value tail along the $x$-axis. The distribution of CARS-converted anti-Stokes pulses should more closely mirror those of the Stokes pulses when the phase matching condition is satisfied.

\section{Discussion}

The extreme value behavior reported in this paper has an intriguing association with power-law behavior observed in social phenomena. Although the number of extreme value pulses is small, their contribution to the total energy cannot be ignored. Pump pulses with slightly larger power than the mean create unproportionally larger gain through the nonlinear transfer function of the gain process. In what can be viewed as the "survival of the fittest (pulses)," the impact of high-energy pulses in the output Stokes distribution is magnified. In fact, we find that $84 \%$ of the pump energy transfer to the Stokes occurs for $16 \%$ of the pulses. Mathematically, the $84 / 16$ ratio is empirically calculated by finding the value of $k$ at which $k \%$ of the pulses carry $(100-k) \%$ of the transferred energy from the pump. This is shown in Fig. 7. The 84/16 ratio offers an intriguing resemblance to the Pareto Principle otherwise known as the " $80 / 20$ rule" which contends that $20 \%$ of the population controls $80 \%$ of the wealth [19]. Similar behavior is also observed in many other phenomena such as geographical distribution of population and casualty losses from natural disasters. This unlikely parallel between nonlinear optics and these seemingly unconnected systems arise because in both cases, the interaction is governed by the transformation of a normal distribution, representing the 


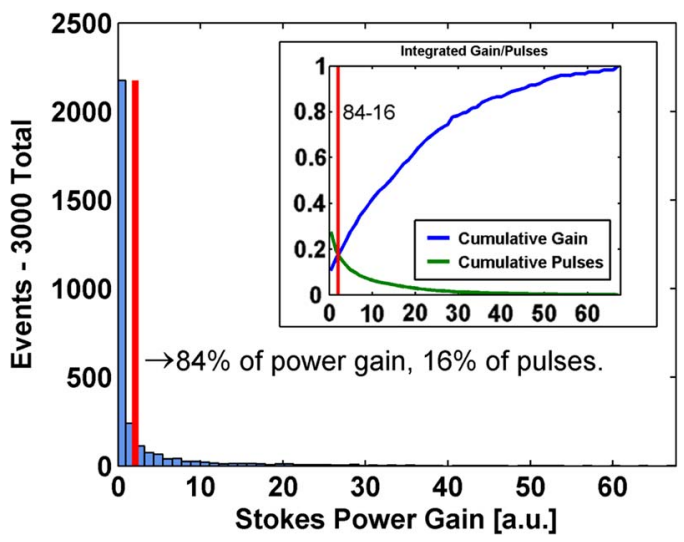

Fig. 7. The figure shows that $84 \%$ of the cumulative pump energy transfer, or gain, occurs in just $16 \%$ of the pulses. The inset shows the normalized cumulative pump energy transfer versus the cumulative pulses accounted for; the intersection is shown by the red line at the $84 / 16$ intersection. Note that $70 \%$ of the cumulative pulses recorded are in the first bin, therefore the pulses curve starts around $30 \%$.

initial condition, by a nonlinear transfer function. Analogously, the work presented here describing SRS extreme events may be extendable to other nonlinear systems recently studied [20], [21]. The environmental conditions, being the initial normal distribution and the form of the nonlinear transfer function, dictate the resulting distribution. We note that the observed 80/20 environmental observation is not a unique or preferred behavior. The initial distribution parameters $\sigma$ and $\nu$ along with the Raman gain parameter $g_{R}$ completely describe the initial and resulting effects which dictate the distribution and implications, in this case the $84 / 16$ ratio. The distribution depends on the amount of fluctuations in the pump laser and the magnitude of the gain which can result in other extreme value forms.

\section{Conclusion}

In summary, we have shown that the fluctuations of Raman amplified Stokes signal and coherent anti-Stokes Raman scattered signal, in the presence of a noisy pump, follow extreme value statistics and have provided mathematical insight into its origin. Our observations have important practical implications. For example, a few Stokes pulses carry most of the converted beam energy. Also, the extreme deviation from Gaussian statistics implies that the traditional characterization based on the standard deviation will provide a highly incomplete description of the pulse-to-pulse fluctuations.

\section{Acknowledgment}

The authors thank Drs. D. Dimitropoulos and D. R. Solli of UCLA for discussions.

\section{References}

[1] D. R. Solli, C. Ropers, P. Koonath, and B. Jalali, "Optical rogue waves," Nature, vol. 450, no. 7172, pp. 1054-1057, Dec. 2007.

[2] J. M. Dudley, G. Genty, and B. J. Eggleton, "Harnessing and control of optical rogue waves in supercontinuum generation," Opt. Express, vol. 16, no. 6, pp. 3644-3651, Mar. 2008.

[3] K. Hammani, C. Finot, J. M. Dudley, and G. Millot, "Optical rogue-wave-like extreme value fluctuations in fiber Raman lasers," Opt. Express, vol. 16, no. 21, pp. 16 467-16 474, Oct. 2008.

[4] A. Efimov and A. J. Taylor, "Supercontinuum generation and soliton timing jitter in SF6 soft glass photonic crystal fibers," Opt. Express, vol. 16, no. 8, pp. 5942-5953, Apr. 2008.

[5] X. Z. Sang, D. Dimitropoulos, B. Jalali, and O. Boyraz, "Influence of pump-to-signal RIN transfer on noise figure in silicon Raman amplifiers," IEEE Photon. Technol. Lett., vol. 20, no. 24, pp. 2021-2023, Dec. 2008.

[6] I. A. Walmsley, M. G. Raymer, T. Sizer, I. N. Duling, and J. D. Kafka, "Stabilization of Stokes pulse energies in the nonlinear regime of stimulated Raman scattering," Opt. Commun., vol. 53, no. 2, pp. 137-140, Feb. 1985. 
[7] M. G. Raymer, I. A. Walmsley, J. Mostowski, and B. Sobolewska, "Quantum theory of spatial and temporal coherence properties of stimulated Raman scattering," Phys. Rev. A, Gen. Phys., vol. 32, no. 1, pp. 332-344, Jul. 1985.

[8] I. A. Walmsley and M. G. Raymer, "Experimental study of the macroscopic quantum fluctuations of partially coherent stimulated Raman scattering," Phys. Rev. A, Gen. Phys., vol. 33, no. 1, pp. 382-390, Jan. 1986.

[9] M. Scalora and J. W. Haus, "Quantum fluctuations and diffraction in stimulated Raman scattering," Opt. Commun., vol. 87, no. 5/6, pp. 267-272, Feb. 1992.

[10] C. Headley, III, and G. P. Agrawal, "Noise characteristics and statistics of picosecond Stokes pulses generated in optical fibers through stimulated Raman scattering," IEEE J. Quantum Electron., vol. 31, no. 11, pp. 2058-2067, Nov. 1995.

[11] E. Landahl, D. Baiocchi, and J. R. Thompson, "A simple analytic model for noise shaping by an optical fiber Raman generator," Opt. Commun., vol. 150, no. 1-6, pp. 339-347, May 1998.

[12] A. Betlej, P. Schmitt, P. Sidereas, R. Tracy, C. Goedde, and J. Thompson, "Increased Stokes pulse energy variation from amplified classical noise in a fiber Raman generator," Opt. Express, vol. 13, no. 8, pp. 2948-2960, Apr. 2005.

[13] B. Jalali, "Making silicon lase," Sci. Amer., vol. 296, no. 2, pp. 58-65, Feb. 2007.

[14] D. Borlaug and B. Jalali, "Extreme value statistics in silicon photonics," presented at the LEOS Annu. Meeting, Newport Beach, CA, Nov. 9-13, 2008, Paper TuU4.

[15] S. O. Rice, "Mathematical analysis of random noise," Bell Syst. Tech. J., vol. 24, pp. 46-156, 1945.

[16] P. L. Liu, L. E. Fencil, J.-S. Ko, I. P. Kaminow, T. P. Lee, and C. A. Burrus, "Amplitude fluctuations and photon statistics of InGaAsP injection lasers,” IEEE J. Quantum Electron., vol. QE-19, no. 9, pp. 1348-1351, Sep. 1983.

[17] A. E. Siegman, Lasers. Sausalito, CA: University Science, 1986.

[18] C. Lafargue, J. Bolger, G. Genty, F. Dias, J. M. Dudley, and B. J. Eggleton, "Direct detection of optical rogue wave energy statistics in supercontinuum generation," Electron. Lett., vol. 45, no. 4, pp. 217-219, Feb. 2009.

[19] Pareto Principle. [Online]. Available: http://en.wikipedia.org/wiki/Pareto_principle

[20] K. Hammani, C. Finot, and G. Millot, "Emergence of extreme events in fiber-based parametric processes driven by a partially incoherent wave," Opt. Lett., vol. 34, no. 8, pp. 1138-1140, Apr. 2009.

[21] M. A. Foster, A. C. Turner, R. Salem, M. Lipson, and A. L. Gaeta, "Broad-band continuous-wave parametric wavelength conversion in silicon nanowaveguides," Opt. Express, vol. 15, no. 20, pp. 12 949-12 958, Oct. 2007. 